\title{
Circ_0074027 binds to EIF4A3 and promotes gastric cancer progression
}

\author{
YULUO WU $^{1}$ and HAIYANG ZHAO ${ }^{2}$ \\ ${ }^{1}$ Department of Oncology, Guangdong Medical University Affiliated Central People's Hospital of Zhanjiang, \\ Zhanjiang, Guangdong 524000; ${ }^{2}$ Department of Oncology, Beijing Zhongguancun Hospital, Beijing 100190, P.R. China
}

Received January 28, 2021; Accepted July 21, 2021

DOI: $10.3892 / \mathrm{ol} .2021 .12965$

\begin{abstract}
Circular RNAs (circRNAs) have been reported to play an important role in the progression of numerous types of human cancer. The aim of the present study was to determine the effects of circRNA_0074027 (circ_0074027) in gastric cancer (GC), and to elucidate the underlying mechanisms of action. For this purpose, the expression of circ_0074027 in GC cell lines was detected using reverse transcription-quantitative PCR. The effects of circ_0074027 on the proliferation and migration of GC cells were investigated using Cell Counting Kit-8 (CCK-8) and Transwell assays, respectively. The Circular RNA Interactome was used to predict that eukaryotic translation initiation factor 4A3 (EIF4A3) could bind to circ_0074027, which was confirmed using an RNA immunoprecipitation assay. The expression and function of EIF4A3 in GC cells were also determined using western blot analysis, as well as CCK-8, colony formation, wound-healing and Transwell assays. The results revealed that circ_0074027 was highly expressed in GC cell lines in the form of a closed loop. In addition, circ_0074027-knockdown inhibited cellular proliferation and motility. Furthermore, EIF4A3 was predicted to be targeted by circ_0074027 and a positive association was identified between them. The overexpression of EIF4A3 reversed the effects of circ_0074027 on the proliferation and motility of GC cells. In conclusion, the findings of the present study demonstrated that circ_0074027 bound to EIF4A3 and promoted the proliferation and migration capacities of GC cells.
\end{abstract}

\section{Introduction}

Gastric cancer (GC) is the fifth most common type of malignancy worldwide, with $>1,000,000$ new cases estimated to occur each year (1). As GC is often diagnosed at

Correspondence to: Dr Haiyang Zhao, Department of Oncology, Beijing Zhongguancun Hospital, 12 Zhongguancun South Road, Haidian, Beijing 100190, P.R. China

E-mail: zhaohaiyangzhy@yeah.net

Key words: circ_0074027, eukaryotic translation initiation factor 4A3, gastric cancer an advanced stage, the associated mortality rate remains at a high level, and as such, the disease ranks as the third leading cause of cancer-related mortality worldwide, corresponding to 784,000 recorded deaths in 2018 (2). In China, GC is the second largest health burden (3). The most important risk factor for GC is Helicobacter pylori infection, and although morbidity rates have decreased, the number of Helicobacter pylori infections is increasing (4). GC incidence rates are currently increasing in young individuals in high-income countries, and as the population ages, more cases of GC are expected in the future (5). Patients with early-stage GC do not exhibit specific symptoms, and metastases are locally advanced or distant by the time of diagnosis (6). GC treatment includes surgery, chemotherapy, radiotherapy and gene therapy; however, the prognosis of patients with GC remains poor, with a median overall survival time of 10-12 months (7). Therefore, it is imperative to identify biomarkers associated with the early diagnosis and underlying mechanisms of GC progression.

Circular RNAs (circRNAs) are single-stranded, covalently closed RNA molecules that are produced as a set of transcripts from pre-mRNAs through a process known as reverse splicing (8). Characterized by their stable presence in tissues and cells, circRNAs are important regulators of gene expression, while dysregulated circRNA expression has been reported in almost all types of cancer (9). circRNA_0074027 (circ_0074027) is considered to exert a cancer-promoting effect; for example, circ_0074027 has been reported to contribute to the progression of non-small cell lung cancer through the microRNA (miRNA/miR)-335-5p-mediated upregulation of cullin 4B expression (10). circ_0074027 has also been shown to be upregulated in non-small cell lung cancer, and functions as a competing endogenous RNA to accelerate cellular proliferation, apoptosis and invasiveness (11). Similarly, there is evidence that circ_0074027 plays an oncogenic role in glioblastoma, promoting the proliferation and invasiveness of glioblastoma cells (12). However, to the best of our knowledge, the role and underlying mechanisms of circ_0074027 in GC are not yet fully understood.

The aim of the present study was to determine the association between the circ_0074027 expression level, and the migration and proliferation capacities of GC cells, as well as the mechanism of action underlying its functions in GC. 


\section{Materials and methods}

Cell lines and culture. The AGS, MKN45, HGC-27 and SNU-5 human GC cell lines, and the normal gastric mucosa cell line, GES-1, were obtained from The Cell Bank of Type Culture Collection of The Chinese Academy of Sciences. The cells were cultured in DMEM (Gibco; Thermo Fisher Scientific, Inc.) supplemented with 10\% FBS (Invitrogen; Thermo Fisher Scientific, Inc.), $1 \%$ penicillin and $1 \%$ streptomycin, in a $37^{\circ} \mathrm{C}$ humidified incubator with $5 \% \mathrm{CO}_{2}$.

Transfection. MKN45 cells were transfected with small interfering (si)RNAs targeting circ_0074027 (si-circ-1, 5'-TAAGCA CCTGGCGCAGGGACT-3'; si-circ-2, 5'-AAGCACCTGGCG CAGGGACTG-3'; $10 \mathrm{nM}$ ), si-negative control (si-NC, 5'-GCA GCTGACTACAGGCAGCGC-3', $10 \mathrm{nM}$ ), the eukaryotic translation initiation factor 4A3 (EIF4A3) overexpression vector (pc-EIF4A3; $10 \mathrm{nM}$ ) or the control vector (pcDNA3.1; $10 \mathrm{nM}$ ). All vectors were purchased from Shanghai GenePharma Co., Ltd., and all transfections were carried out using Lipofectamine ${ }^{\circledR}$ RNAiMAX (Invitrogen; Thermo Fisher Scientific, Inc.) according to the manufacturer's protocol. Following incubation at $37^{\circ} \mathrm{C}\left(5 \% \mathrm{CO}_{2}\right)$ for $6 \mathrm{~h}$, the culture medium was replaced with fresh medium containing 10\% FBS. The culture was continued in the original environment, and subsequent experimentation was conducted within $24 \mathrm{~h}$.

$R N A$ extraction and reverse transcription-quantitative $(R T-q)$ $P C R$. Total cellular RNA was isolated using TRIzol ${ }^{\circledR}$ reagent (Invitrogen; Thermo Fisher Scientific, Inc.), and reverse transcribed into cDNA using the PrimeScript ${ }^{\mathrm{TM}}$ 1st Strand cDNA Synthesis Kit (Takara Biotechnology Co., Ltd.) according to the manufacturer's protocol. The reaction conditions for reverse transcription were as follows: $95^{\circ} \mathrm{C}$ for $15 \mathrm{sec}, 60^{\circ} \mathrm{C}$ for $1 \mathrm{~min}$ and $72^{\circ} \mathrm{C}$ for $10 \mathrm{~min}, 35$ cycles in total. Subsequently, qPCR was using iTaq $^{\mathrm{TM}}$ Universal SYBR ${ }^{\circledR}$ Green Supermix (Bio-Rad Laboratories, Inc.) on an ABI 7500 system (Applied Biosystems; Thermo Fisher Scientific, Inc.) using the following thermocycling conditions: Initial denaturation at $95^{\circ} \mathrm{C}$ for $10 \mathrm{~min}$; followed by 40 cycles of $95^{\circ} \mathrm{C}$ for $10 \mathrm{sec}, 60^{\circ} \mathrm{C}$ cycles for $20 \mathrm{sec}$ and $70^{\circ} \mathrm{C}$ cycles for $30 \mathrm{sec}$. The relative expression of genes was calculated using the $2^{-\Delta \Delta \mathrm{Cq}}$ method (13). with GAPDH expression as the internal control for normalization. The sequences of the primers are as follows: circ_0074027 forward, 5'-GCGTCCCTGTGTATGTTGGA-3' and reverse, 5'-GTCTGTCTTAAAGCGACAGCG-3'; paired like homeodomain 1 (PITX1) forward, 5'-GCTACCCCGACATGA GCA-3' and reverse, 5'-GTTACGCTCGCGCTTACG-3'); and GAPDH forward, 5'-GTGGGCATCAATGGATTTGG-3' and reverse, 5'-ACACCATGTATTCCGGGTCAAT-3'.

RNase R digestion. A total of $10 \mathrm{U}$ RNase $\mathrm{R}$ (Geneseed Biotech Co., Ltd.) was added to $2.5 \mu \mathrm{g}$ total RNA extracted from MKN45 cells, and incubated at $37^{\circ} \mathrm{C}$ for $30 \mathrm{~min}$, followed by RT-qPCR to determine the expression levels of circ_0074027 and PITX1.

Cell counting kit-8 (CCK-8) assay. MKN45 cells were seeded into 96 -well plates $\left(5 \times 10^{3}\right.$ cells/well) and incubated at $37^{\circ} \mathrm{C}$ for 0, 24, 48 and $72 \mathrm{~h}$, following the addition of si-circ_0074027 or co-transfection with pc-EIF4A3. Next, $10 \mu$ l CCK-8 reagent (Beijing Solarbio Science \& Technology Co., Ltd.) was added to each well and incubated for a further $2.5 \mathrm{~h}$ at $37^{\circ} \mathrm{C}$. The absorbance was measured at a wavelength of $450 \mathrm{~nm}$ using a microplate reader (SPECTROstar Nano; BMG Labtech $\mathrm{GmbH})$.

Colony formation assay. MKN45 cells were seeded into 6 -well plates $\left(1 \times 10^{3}\right.$ cells/well) and cultured in DMEM at $37^{\circ} \mathrm{C}$ for 10 days, replacing the medium every 3 days. Subsequently, the cells were fixed with $4 \%$ paraformaldehyde for $30 \mathrm{~min}$, stained with $0.5 \%$ crystal violet (Beijing Solarbio Science \& Technology Co., Ltd.) for $10 \mathrm{~min}$ (both at room temperature), and the number of colonies with $>50$ cells was manually counted using a light microscope (Olympus Corporation).

Wound-healing assay. MKN45 cells were seeded into 6-well culture plates $\left(2 \times 10^{5}\right.$ cells/well $)$ and cultured at $37^{\circ} \mathrm{C}$ until reaching $90 \%$ confluency. The monolayer was then scratched with a sterile pipette tip, and the cells were cultured in serum-free medium (Beijing Solarbio Science \& Technology Co., Ltd.). Wound distances were photographed after 0 and $48 \mathrm{~h}$ using a light microscope (magnification, x100; Olympus Corporation).

Transwell assay. Transfected cells $\left(1 \times 10^{4}\right.$ cells/well $)$ were seeded into the upper chamber of a Transwell insert pre-coated with Matrigel ${ }^{\circledR}$ (Corning, Inc.) in serum-free medium. Complete medium containing $10 \%$ FBS was added to the lower chamber. Following a 12 -h incubation at $37^{\circ} \mathrm{C}$, residual cells on the surface were removed, and the migratory cells were fixed with $4 \%$ paraformaldehyde, followed by staining with $0.1 \%$ crystal violet for $15 \mathrm{~min}$ at $37^{\circ} \mathrm{C}$. The migration level was observed by counting the cells under a light microscope (magnification, x100; Olympus Corporation).

RNA binding immunoprecipitation (RIP) assay. The Magna RIP RNA Binding Protein Immunoprecipitation kit (Product ID: RIP; MilliporeSigma) was used for RIP, according to the manufacturer's instructions. MKN45 cell lysates were collected and $100 \mu 1$ lysate per reaction was incubated with NC IgG (1:500; cat. no. ab172730) or anti-EIF4A3 (1:1,000; cat. no. ab180573) antibody (both Abcam) conjugated to protein $\mathrm{A} / \mathrm{G}$ magnetic beads (EMD Millipore) at $4^{\circ} \mathrm{C}$ overnight. Immunoprecipitated RNA was then extracted and detected by RT-qPCR to confirm the enrichment of binding targets.

Protein isolation and western blot analysis. Total cellular proteins in MKN45 cells were extracted using RIPA lysis buffer (Beyotime Institute of Biotechnology) and the protein concentration was determined using a BCA protein assay kit (Beyotime Institute of Biotechnology). Proteins (30 ng per lane) were separated via $10 \%$ SDS-PAGE, after which the samples were transferred to a polyvinylidene difluoride membrane (Beyotime Institute of Biotechnology). The membranes were blocked with 5\% skimmed milk for $2 \mathrm{~h}$ at room temperature, followed by an overnight incubation at $4{ }^{\circ} \mathrm{C}$ with the primary antibodies. Following the primary antibody incubation, the membranes were incubated with a goat anti-mouse secondary 
A

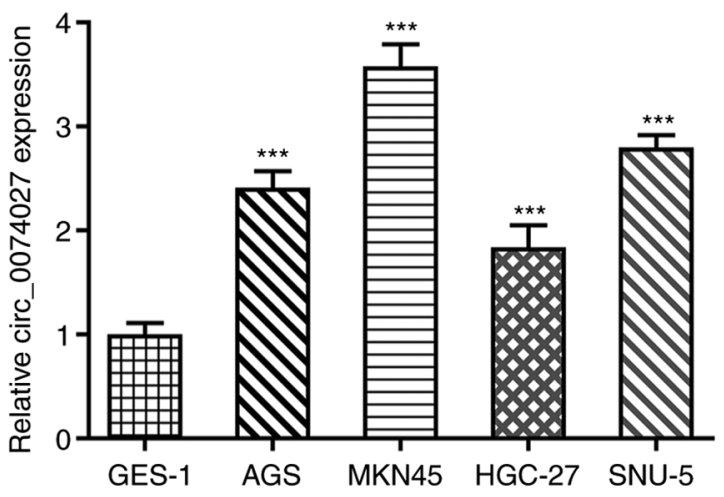

B

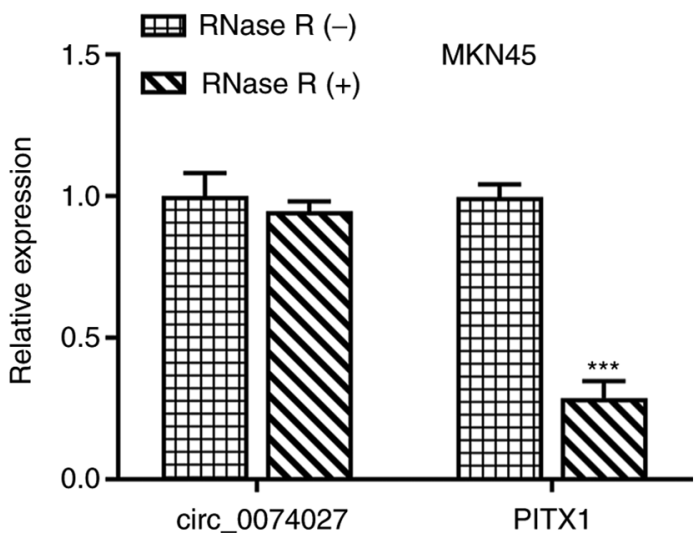

Figure 1. Expression levels of circ_0074027 are increased in gastric cancer. (A) RT-qPCR was performed to quantify circ_0074027 expression in GES-1, AGS, MKN45, HGC-27 and SNU-5 cells. (B) Expression levels of circ_0074027 and PITX1 in MKN45 cells with or without RNase R treatment were detected using RT-qPCR. ${ }^{* * *}$ P<0.001 vs. GES-1 or RNase (-). RT-qPCR, reverse transcription-quantitative PCR; circ_0074027, circular RNA_0074927; PITX1, paired like homeodomain 1 .

antibody (1:5,000; cat. no. S0001; Affinity Biosciences,) for $2 \mathrm{~h}$ at room temperature. Signals were visualized using an ECL kit (Beyotime Institute of Biotechnology, Inc.) and ImageJ software (v1.8.0; National Institutes of Health) was used for densitometric analysis. The primary antibodies were as follows: Anti-EIF4A3 (1:1,000; cat. no. ab180573; Abcam) and anti-GAPDH (1:2,500; cat. no. ab9485; Abcam).

Bioinformatics analysis. To further elucidate its potential mechanism in GC, the Circular RNA Interactome (https:/circinteractome.irp.nia.nih.gov/) was used to predict the factors which bound to circ_0074027. In order to analyze the overall level of differential expression of EIF4A3 in normal subjects and patients with stomach adenocarcinoma, clinical data were obtained from the Gene Expression Profile Interaction Analysis (GEPIA) database (http:/gepia. cancer-pku.cn/). The database has definitive online genomic analysis technology based on large-scale sequencing, while omitting information that may identify patients.

Statistical analysis. Data are presented as the mean \pm SD of at least three independent experiments. Significant differences between groups were determined using an independent unpaired t-test or one-way ANOVA followed by Tukey's post hoc test. Statistical analyses were performed using GraphPad Prism 6 software (GraphPad Software, Inc.), and $\mathrm{P}<0.05$ was considered to indicate a statistically significant difference.

\section{Results}

Circ_0074027 is highly expressed in GC cell lines. To investigate the expression level of circ_0074027, GC cell lines (AGS, MKN45, HGC-27 and SNU-5) and GES-1 cells were subjected to RT-qPCR. As shown in Fig. 1A, compared with GES-1 cells, circ_0074027 was highly expressed in the GC cell lines, particularly in MKN45 cells. Thus, MKN45 cells were selected for use in subsequent experiments. RNase R digestion assays subsequently revealed that linear PITX1 (as the paired linear form of circ_0074027), could be digested by RNase R. However, circ_0074027 was resistant to RNase R digestion in MKN45 cells (Fig. 1B), suggesting that circ_0074027 features a closed-loop structure. These results indicated that circ_0074027 expression was upregulated in GC cell lines.

Circ_0074027-knockdown inhibits the proliferation and motility of $G C$ cells. In order to investigate the biological function of circ_0074027 in GC, MKN45 cells were transfected with si-circ_0074027. The results of RT-qPCR analysis revealed that the knockdown efficiency of si-circ-1 was greater than that of si-circ-2 (Fig. 2A). As revealed by CCK-8 analysis, the proliferative capacity of MKN45 cells was significantly inhibited following circ_0074027 silencing, compared with the si-NC group, with a more pronounced effect observed with si-circ-1 (Fig. 2B). Similarly, the results of the colony formation assay indicated that circ_0074027-knockdown markedly decreased the colony formation ability of MKN45 cells, compared with the si-NC group, with fewer colonies observed following transfection with si-circ-1 than with si-circ-2 (Fig. 2C). The results of Transwell and wound-healing assays revealed the role of circ_0074027 in the migration of GC cells. Both the si-circ-1 and si-circ-2 groups exhibited fewer positively-stained cells (Fig. 2D), suggesting that the silencing of circ_0074027 suppressed the invasive and migration abilities of MKN45 cells. After 48 h, circ_0074027-knockdown markedly delayed wound closure compared with the si-NC group (Fig. 2E). These data demonstrated that the circ_0074027 expression level was closely associated with the proliferation and migration of GC cells, and that the reduction in circ_0074027 expression exerted an anti-oncogenic effect.

Silencing of circ_0074027 leads to the downregulation of EIF4A3 expression. To further elucidate the potential mechanisms of circ_0074027 in GC, the Circular RNA Interactome was used to predict factors that bound to EIF4A3 (Fig. 3A). An RIP assay was also performed to confirm the prediction; circ_0074027 was found to be abundantly enriched with EIF4A3 in MKN45 cells, relative to the NC IgG (Fig. 3B). Furthermore, the GEPIA results revealed higher expression of EIF4A3 in patients with stomach adenocarcinoma, compared with healthy individuals (Fig. 3C). Therefore, it 

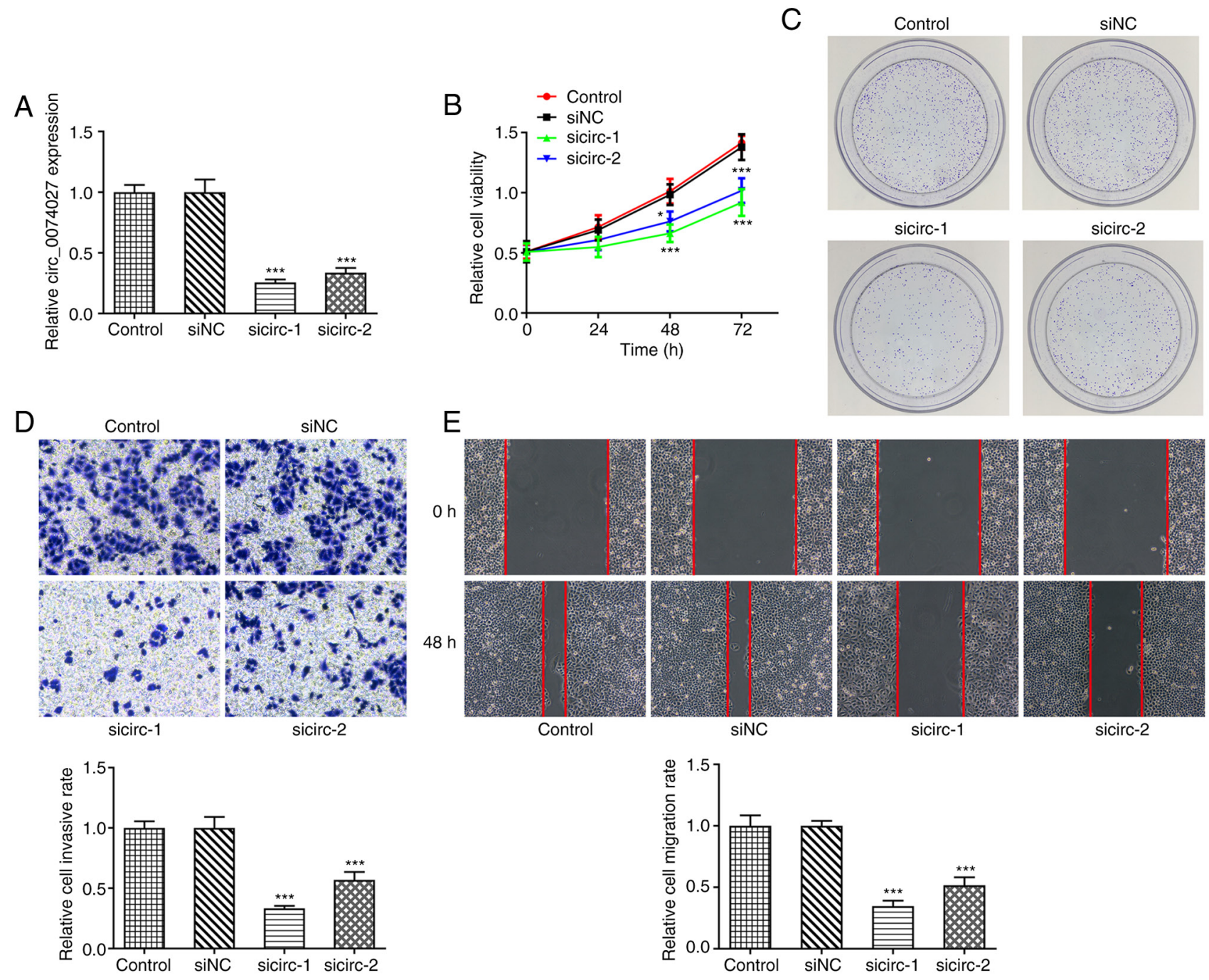

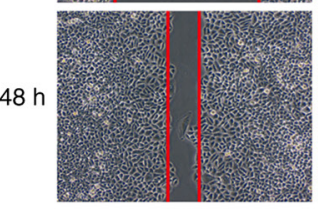

Control

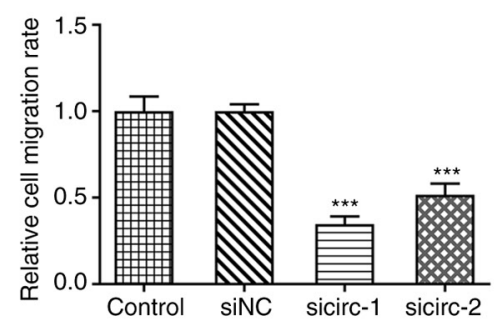

Figure 2. Knockdown of circ_0074027 impedes gastric cancer cell proliferation and motility. MKN45 cells were transfected with si-NC, si-circ-1 or si-circ-2. (A) Expression of circ_0074027 was detected using RT-qPCR following transfection. (B) Viability of MKN45 cells was assessed using a Cell Counting Kit-8 assay. (C) Colony formation capacity of MKN45 cells following transfection was assessed using a colony formation assay. Migratory ability of MKN45 cells following transfection was analyzed using (D) Transwell and (E) wound-healing assays (magnification, x100). $\mathrm{P}<0.05{ }^{* * * *} \mathrm{P}<0.001 \mathrm{vs}$. siNC. RT-qPCR, reverse transcription-quantitative PCR; circ_0074027, circular RNA_0074927; sicirc, small interfering RNA of circ_0074027; si, small interfering RNA; $\mathrm{NC}$, negative control.

was hypothesized that circ_0074027 may play a role in GC by binding to EIF4A3. Subsequently, the expression of EIF4A3 in GC cell lines was analyzed by RT-qPCR. The results revealed that EIF4A3 was more highly expressed in GC cell lines compared with GES-1 cells, with the highest expression observed in MKN45 cells (Fig. 3D). Following transfection with si-circ_0074027, the expression of EIF4A3 in MKN45 cells was decreased, and si-circ-1 was found to exert a more prominent effect (Fig. 3E). These results indicated that circ_0074027 was positively associated with EIF4A3 in GC.

Overexpression of EIF4A3 attenuates the suppressive effects of circ_0074027 on GC cell proliferation. To further determine the role of circ_0074027 and EIF4A3 in GC, the EIF4A3 overexpression plasmid (pc-EIF4A3) was used in the following experiments. The results of western blot analysis revealed that pc-EIF4A3 significantly elevated the expression of EIF4A3, compared with pcDNA3.1 (Fig. 4A). As revealed by CCK- 8 analysis, MKN45 cells co-transfected with si-circ-1 and pc-EIF4A3 exhibited greater proliferative ability than those co-transfected with si-circ-1 and pcDNA3.1 (Fig. 4B). The results of the colony formation assay indicated that the colony-forming ability of MKN45 cells was decreased following circ_0074027-knockdown, compared with the control, while it was significantly restored following co-transfection with pc-EIF4A3 (Fig. 4C). Collectively, these findings indicated that elevated EIF4A3 expression reversed the inhibitory effects of circ_0074027 on the proliferation of GC cells.

Overexpression of EIF4A3 counteracts the inhibitory effects on motility generated by circ_0074027-knockdown. Further experiments were conducted to examine the effects of circ_0074027 and EIF4A3 on the motility of GC cells. Both Transwell and wound-healing assays (Fig. 5A and B) revealed that circ_0074027-knockdown inhibited MKN45 cell migration, and that the overexpression of EIF4A3 attenuated this effect. The si-circ-1 + pcDNA3.1 group did not exhibit 
A

\begin{tabular}{|c|c|c|c|}
\hline CircRNA ID & hsa_circ_0074027 & Location & $\frac{\mathrm{chr} 5: 134363423-}{134369964}$ \\
\hline $\begin{array}{l}\text { Genomic } \\
\text { Length }\end{array}$ & $6541 \mathrm{bp}$ & $\begin{array}{l}\text { Spliced Seq } \\
\text { Length }\end{array}$ & 2383 bp \\
\hline Best Transcript & NM 002653 Primers & Gene Symbol & $\underline{P I T X 1}$ \\
\hline Samples & A549 & Study & Salzman2013 \\
\hline GenomicSeq & hsa_circ 0074027 & Mature Seq & hsa_circ_0074027 \\
\hline \multicolumn{4}{|c|}{ RNA-binding protein sites matching to circRNAs } \\
\hline \multicolumn{4}{|c|}{ RNA-binding protein sites matching flanking regions of circRNA } \\
\hline \multicolumn{3}{|c|}{ RNA-binding Protein } & \# Tags \\
\hline \multicolumn{3}{|c|}{ DGCR8 } & 2 \\
\hline \multicolumn{3}{|c|}{ EIF4A3 } & 7 \\
\hline
\end{tabular}

$\mathrm{D}$
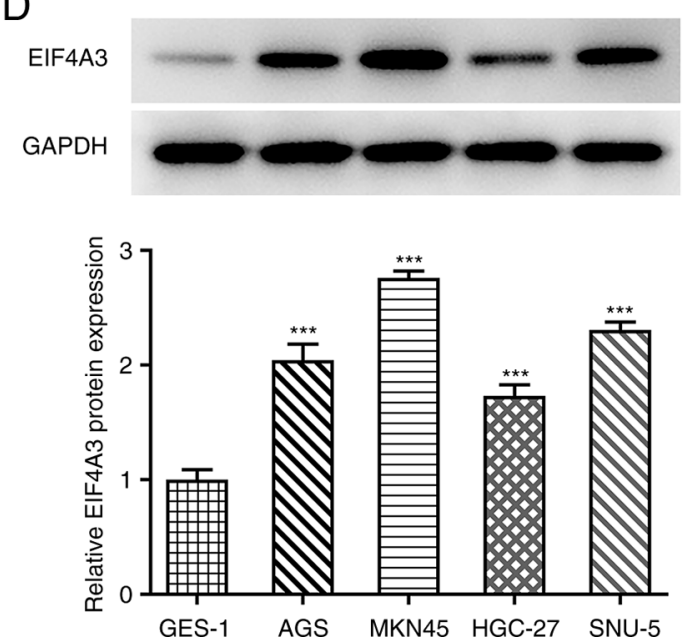

B

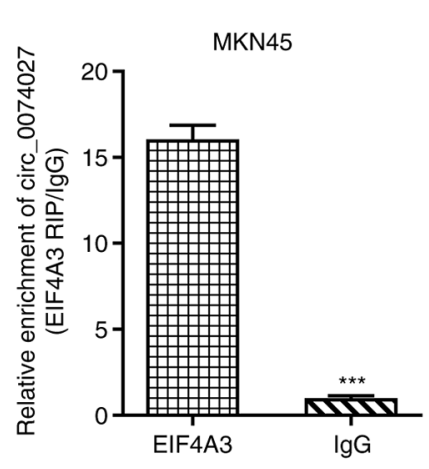

$\mathrm{E}$
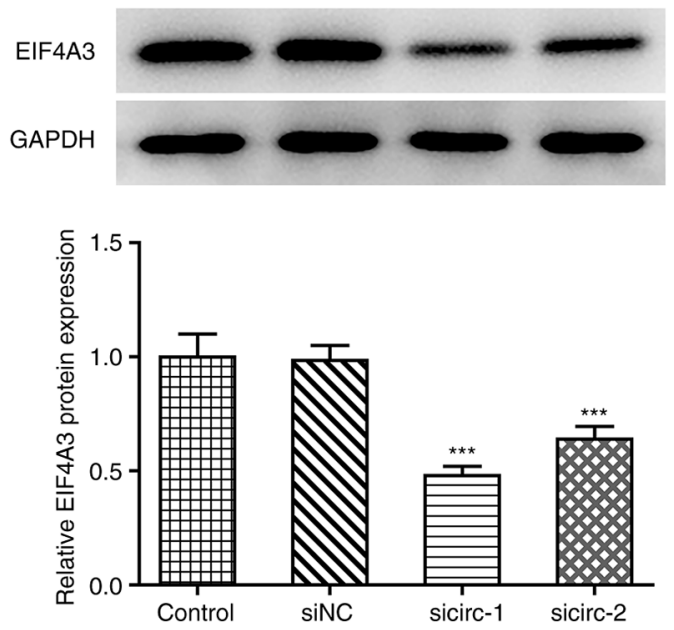

Figure 3. circ_0074027 is positively associated with EIF4A3. (A) Circular RNA Interactome analysis predicted genes that bound to circ_0074027. (B) circ_0074027 with EIF4A3 enrichment, which was measured using an RNA immunoprecipitation assay. (C) Gene expression of EIF4A3 profiled across tumor samples and tissues from normal patients, acquired from the Gene Expression Profiling Interactive Analysis database. (D) Expression of EIF4A3 was detected using RT-qPCR in gastric cancer cells following transfection. (E) Expression of EIF4A3 was detected using RT-qPCR in MKN45 cells following transfection with si-NC, si-circ-1 or si-circ-2. ${ }^{*} \mathrm{P}<0.05$ and ${ }^{* * * *} \mathrm{P}<0.001$ vs. GES-1 or siNC. RT-qPCR, reverse transcription-quantitative PCR; circ_0074027, circular RNA_0074927; sicirc, small interfering RNA of circ_0074027; si, small interfering RNA; NC, negative control; EIF4A3, eukaryotic translation initiation factor 4A3; STAD, stomach adenocarcinoma.

any marked differences compared with the si-circ-1 group, whereas the si-circ-1 + pc-EIF4A3 group exhibited a markedly enhanced migration rate. These results demonstrated that the elevated expression of EIF4A3 counteracted the inhibitory effects of circ_0074027-knockdown on the motility of GC cells.

\section{Discussion}

CircRNAs, a newly identified group of non-coding RNAs, have gained increasing attention in the field of cancer research (14). Emerging evidence has indicated the existence of thousands of endogenous circRNAs in mammalian cells, which can modulate gene expression by binding to miRNAs or other molecules, and suppressing their function at the transcriptional or post-transcriptional level (15). In the present study, silencing circ_0074027 was demonstrated to inhibit the proliferation and migration of GC cells, and this effect was reversed by the overexpression of EIF4A3.

Firstly, circRNAs were found to be present in GC cells in the form of a closed loop, and to be highly expressed therein. Based on previous studies, this result is in accordance with the functions of circRNAs in tumors. For example, circ_0067934 was found to be expressed at higher levels in esophageal cancer compared with normal tissues (16). In addition, the expression level of circ_002059 in the plasma of patients with post-operative GC has been shown to be higher than that of patients with pre-operative GC (17). Moreover, circ_0005075 has been shown to be aberrantly expressed in hepatocellular carcinoma tissues (18). Previously, Qian et al (12) revealed that circ_0074027 upregulation promoted glioblastoma cell proliferation and invasiveness. Subsequently, circ_0074027 has been reported to promote the progression of non-small cell lung cancer (10). For example, Duan et al (19) revealed that circ_0074027 contributed to non-small cell lung cancer progression by positively modulating RHOA via miR-2467-3p sequestration. Furthermore, Jiang et al (20) demonstrated that circ_0074027 promoted the progression of non-small cell lung cancer via the miR-362-3p/clathrin heavy chain axis. Based on the aforementioned research conclusions, the present study confirmed that the overexpression of circ_0074027 had a potential promoting effect on cancer, such as enhancing cancer cell proliferation and migration. Then, the Circular RNA Interactome was used to predict that EIF4A3 was 
A
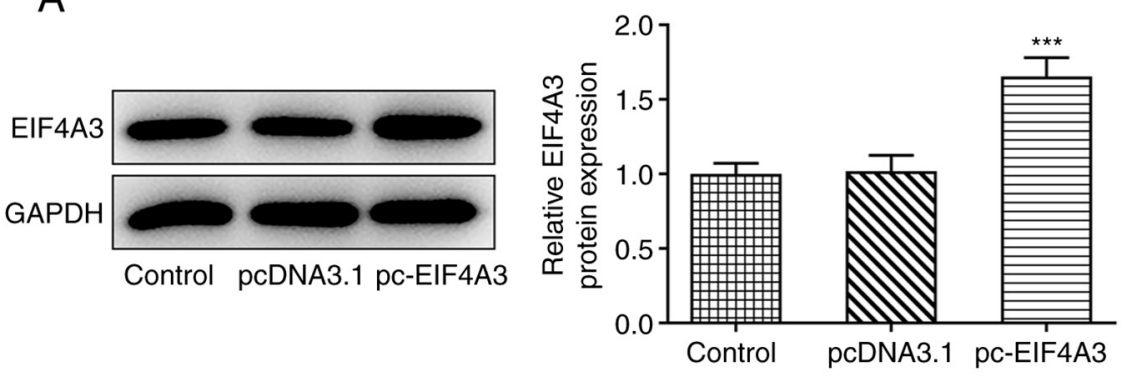

C
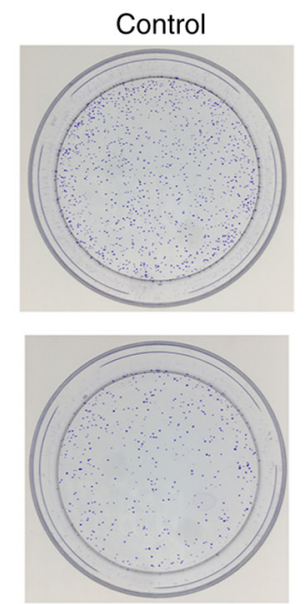

sicirc-1+pcDNA3.1
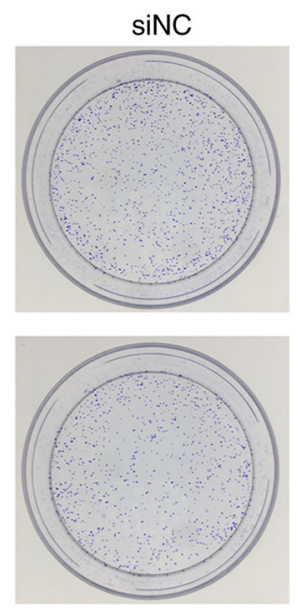

sicirc-1+pc-EIF4A3
B

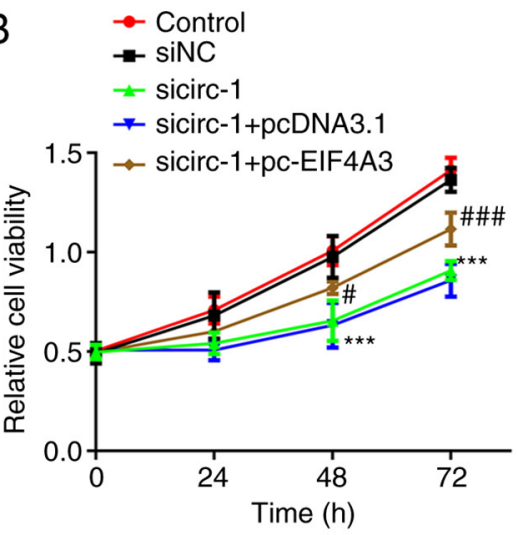

Figure 4. Overexpression of EIF4A3 reverses the suppressive effects of circ_0074027 silencing on gastric cancer cell proliferation. MKN45 cells were transfected with pcDNA3.1, pc-EIF4A3 and si-circ-1. (A) Expression of EIF4A3 was detected using western blot analysis in MKN45 cells following transfection. (B) Viability of MKN45 cells was assessed using a Cell Counting Kit-8 assay. (C) Colony numbers of MKN45 cells following transfection were determined using a colony formation assay. ${ }^{* * *} \mathrm{P}<0.001$ vs. siNC; ${ }^{\#} \mathrm{P}<0.001$ and ${ }^{\# \# \#} \mathrm{P}<0.001$ vs. sicirc-1 + pcDNA3.1. EIF4A3, eukaryotic translation initiation factor 4A3; circ_0074027, circular RNA_0074927; sicirc, small interfering RNA of circ_0074027; si, small interfering RNA; NC, negative control.

able to bind circ_0074027. Furthermore, the expression of EIF4A3 was found to be higher in GC. Thus, the present study aimed to determine the biological function of circ_0074027 in GC. The proliferation and migration of tumor cells affect the malignant progression of tumors (21); thus, the effect of f circ_0074027-knockdown on GC cells was analyzed using CCK-8, colony formation, Transwell and wound-healing assays. Silencing of circ_0074027 was found to inhibit the progression of GC cells. This is consistent with the findings of previous studies, which identified that circ_0074027 functioned as a tumor promoter in GC cells (22). And circ_0074027 has also been shown to contribute to the progression of GC (22).

In order to elucidate the potential mechanisms of circ_0074027 in GC, the Circular RNA Interactome database was used to screen for the gene with the most tags for RNA binding, EIF4A3. A previous study implicated EIF4A3 as an important component of RNA splicing (23). Moreover, as an RNA binding protein, EIF4A3 regulates the expression of non-coding RNAs in tumors (24), and bioinformatics analysis revealed that EIF4A3 expression was upregulated at the transcriptional level in common malignant tumors, such as breast, lung and urinary cancers (25). In the present study, the binding of circ_0074027 to EIF4A3 was confirmed using an RIP assay. The expression of EIF4A3 in GC was then verified through
GEPIA, and GC cell lines expressing high levels of EIF4A3. Notably, EIF4A3 was found to be positively associated with circ_0074027. EIF4A3 has been shown to trigger the proliferation, invasion and metastasis of glioblastoma multiforme cells, and thus contributes to tumorigenesis (26). It has also been demonstrated that E2F transcription factor 1 and EIF4A3 coordinate with circSEPT9 to facilitate the development and progression of triple-negative breast cancer through the circSEPT9/miR-637/LIF IL6 family cytokine axis (27). Similarly, the results of the present study confirmed the carcinogenic effect of EIF4A3. The increased expression of EIF4A3 counteracted the inhibitory effects of circ_0074027-knockdown on the proliferation and motility of GC cells. Collectively, the present study provides a novel insight into the functions of circ_0074027 in GC, which are hypothesized to occur via the regulation of EIF4A3. However, the study had certain limitations. For example, only in vitro experiments were performed, and tissue samples were not used for validation. In addition, the molecular mechanism of EIF4A3 in tumor progression remains unclear. Thus, future studies are required to further elucidate the mechanisms involved.

In conclusion, the findings of the present study indicated that the expression of circ_0074027 and EIF4A3 were upregulated in GC cells. circ_0074027 modulated GC cell proliferation 
A

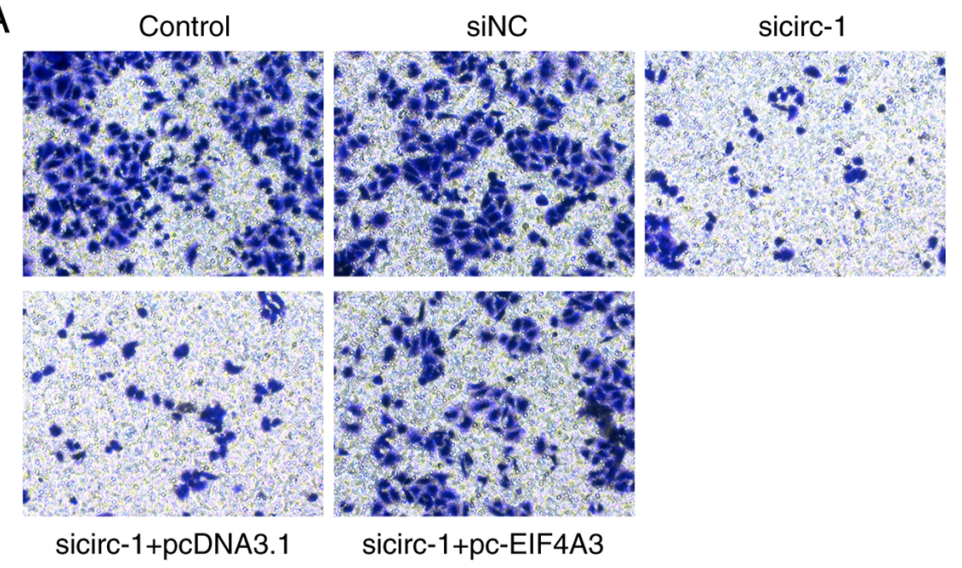

B

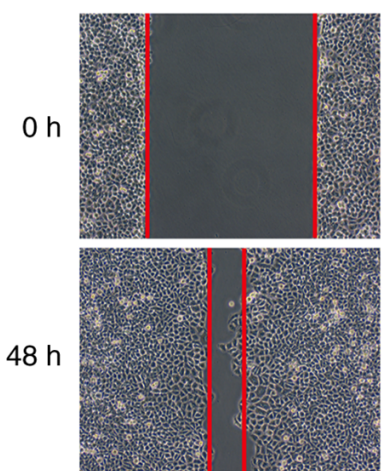

Control
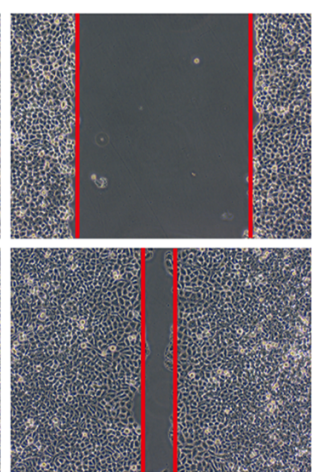

siNC
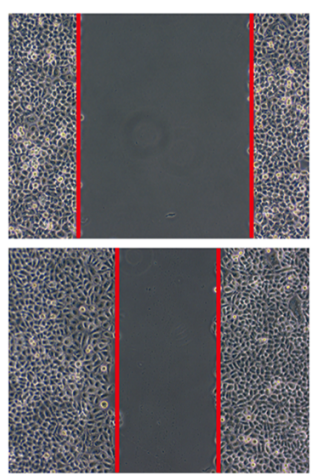

sicirc-1
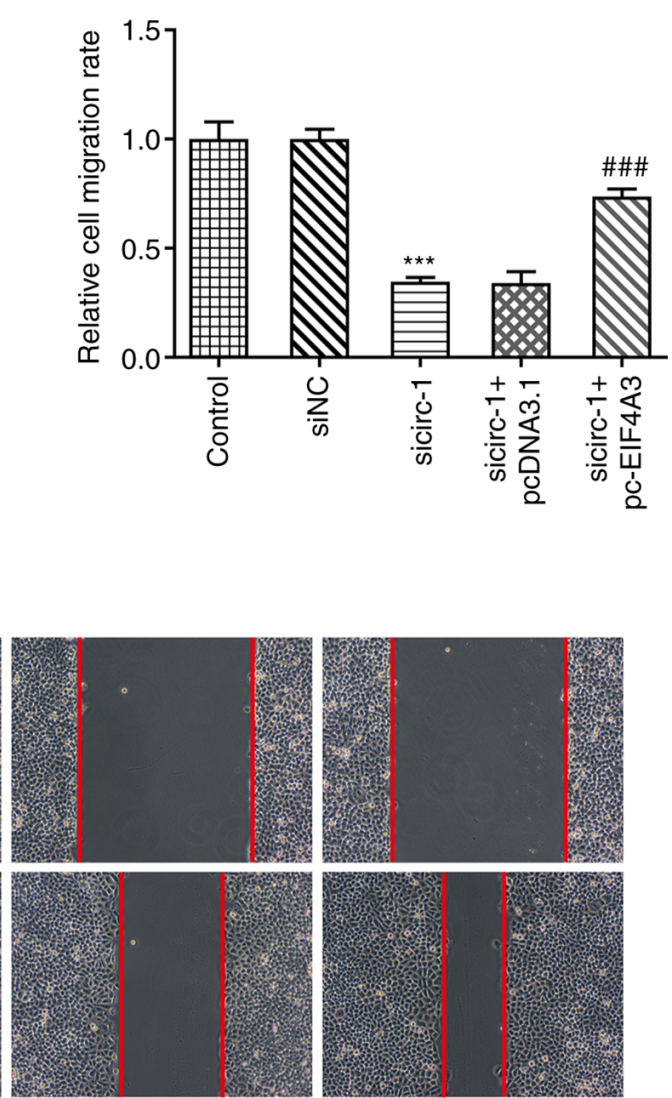

sicirc-1+pcDNA3.1
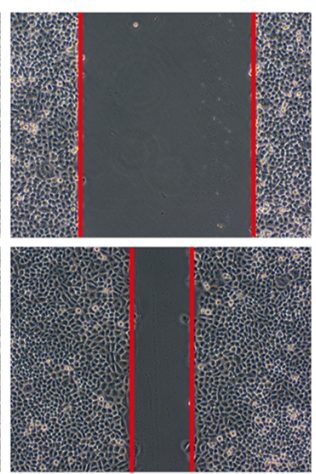

sicirc-1+pc-EIF4A3

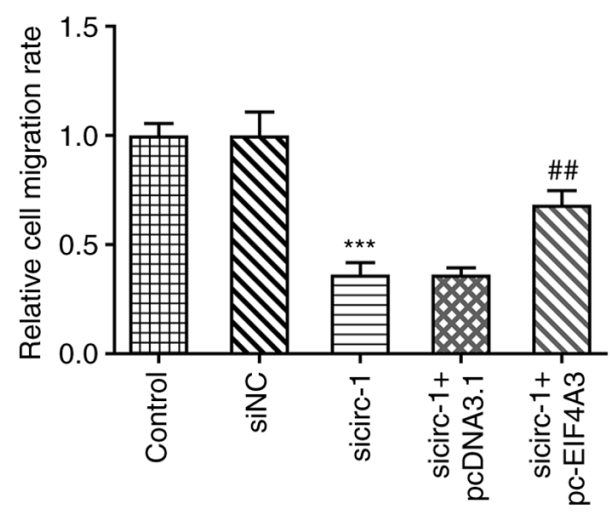

Figure 5. Overexpression of EIF4A3 counteracts the inhibitory effects of circ_0074027 silencing on gastric cancer cell motility. (A) Migratory ability of MKN45 cells following transfection was analyzed by Transwell assay (magnification, x100). (B) Migratory ability of MKN45 cells following transfection was analyzed using a wound-healing assay (magnification, $\mathrm{x} 100) .{ }^{* * *} \mathrm{P}<0.001 \mathrm{vs}$. siNC; ${ }^{\# \prime} \mathrm{P}<0.01$, ${ }^{\# \# \#} \mathrm{P}<0.001 \mathrm{vs}$. sicirc-1 + pcDNA3.1. EIF4A3, eukaryotic translation initiation factor 4A3; circ_0074027, circular RNA_0074927; sicirc, small interfering RNA of circ_0074027; si, small interfering RNA.

and migration through targeted binding of EIF4A3. This finding may help to elucidate the molecular mechanisms of GC progression. In addition, circ_0074027 may prove to be a promising prognostic biomarker for GC and a novel candidate for targeted GC therapy.

\section{Acknowledgements}

Not applicable.

\section{Funding}

No funding was received.

\section{Availability of data and materials}

The datasets used and/or analyzed during the current study are available from the corresponding author on reasonable request.

\section{Authors' contributions}

YW and HZ conceived and designed the study, acquired and interpreted the data. YW was a major contributor in writing the manuscript. YW and $\mathrm{HZ}$ confirm the authenticity of all the raw data. Both authors have read and approved the final manuscript. 


\section{Ethics approval and consent to participate}

Not applicable.

\section{Patient consent for publication}

Not applicable.

\section{Competing interests}

The authors declare that they have no competing interests.

\section{References}

1. Smyth EC, Nilsson M, Grabsch HI, van Grieken NC and Lordick F: Gastric cancer. Lancet 396: 635-648, 2020.

2. Johnston FM and Beckman M: Updates on management of gastric cancer. Curr Oncol Rep 21: 67, 2019.

3. Chen W, Zheng R, Baade PD, Zhang S, Zeng H, Bray F, Jemal A, Yu XQ and He J: Cancer statistics in China, 2015. CA Cancer J Clin 66: 115-132, 2016.

4. Lee YC, Chiang TH, Chou CK, Tu YK, Liao WC, Wu MS and Graham DY: Association between Helicobacter pylori eradication and gastric cancer incidence: A systematic review and meta-analysis. Gastroenterology 150: 1113-1124.e5, 2016.

5. Venerito M, Link A, Rokkas T and Malfertheiner P: Gastric cancer-clinical and epidemiological aspects. Helicobacter 21 (Suppl 1): S39-S44, 2016.

6. Yu J, Huang C, Sun Y, Su X, Cao H, Hu J, Wang K, Suo J, Tao K, He X, et al: Effect of laparoscopic vs open distal gastrectomy on 3-year disease-free survival in patients with locally advanced gastric cancer: The CLASS-01 randomized clinical trial. JAMA 321: 1983-1992, 2019.

7. Oliveira C, Pinheiro H, Figueiredo J, Seruca R and Carneiro F: Familial gastric cancer: Genetic susceptibility, pathology, and implications for management. Lancet Oncol 16: e60-e70, 2015.

8. Capel B, Swain A, Nicolis S, Hacker A, Walter M, Koopman P, Goodfellow P and Lovell-Badge R: Circular transcripts of the testis-determining gene Sry in adult mouse testis. Cell 73: 1019-1030, 1993

9. Vo JN, Cieslik M, Zhang Y, Shukla S, Xiao L, Zhang Y, Wu YM, Dhanasekaran SM, Engelke CG, Cao X, et al: The landscape of circular RNA in cancer. Cell 176: 869-881.e13, 2019.

10. Yu C, Ying J, Yu K, Shen W and Jiang M: Circ 0074027 contributes to nonsmall cell lung cancer progression by upregulating CUL4B expression through miR-335-5p. Cancer Biother Radiopharm: Jun 22, 2020 (Epub ahead of print).

11. Gao P, Wang Z, Hu Z, Jiao X and Yao Y: Circular RNA circ 0074027 indicates a poor prognosis for NSCLC patients and modulates cell proliferation, apoptosis, and invasion via miR-185-3p mediated BRD4/MADD activation. J Cell Biochem 121: 2632-2642, 2020.

12. Qian L, Guan J, Wu Y and Wang Q: Upregulated circular RNA circ 0074027 promotes glioblastoma cell growth and invasion by regulating miR-518a-5p/IL17RD signaling pathway. Biochem Biophys Res Commun 510: 515-519, 2019.
13. Livak KJ and Schmittgen TD: Analysis of relative gene expression data using real-time quantitative PCR and the 2(-Delta Delta C(T)) method. Methods 25: 402-408, 2001.

14. Yu T, Wang Y, Fan Y, Fang N, Wang T, Xu T and Shu Y: CircRNAs in cancer metabolism: A review. J Hematol Oncol 12: 90, 2019.

15. Zhang HD, Jiang LH, Sun DW, Hou JC and Ji ZL: CircRNA: A novel type of biomarker for cancer. Breast Cancer 25: 1-7, 2018.

16. Xia W, Qiu M, Chen R, Wang S, Leng X, Wang J, Xu Y, Hu J, Dong G, Xu PL and Yin R: Circular RNA has_circ_0067934 is upregulated in esophageal squamous cell carcinoma and promoted proliferation. Sci Rep 6: 35576, 2016.

17. Li P, Chen S, Chen H, Mo X, Li T, Shao Y, Xiao B and Guo J: Using circular RNA as a novel type of biomarker in the screening of gastric cancer. Clin Chim Acta 444: 132-136, 2015.

18. Yang X, Song H, Zi Z, Kou J, Chen S, Dai Y, Wang J, Yuan L and Gao K: Circ_0005075 promotes hepatocellular carcinoma progression by suppression of microRNA-335. J Cell Physiol 234: 21937-21946, 2019.

19. Duan Z, Wei S and Liu Y: Circ_0074027 contributes to non-small cell lung cancer progression through positively modulating RHOA via sequestering miR-2467-3p. J Bioenerg Biomembr 53: 223-233, 2021.

20. Jiang Z, Yin J, Peng G and Long X: Circ_0074027 contributes to the progression of non-small cell lung cancer via microRNA-362-3p/clathrin heavy chain axis. Anticancer Drugs 32: $1-10,2021$

21. Xue YF, Li M, Li W, Lin Q, Yu BX, Zhu QB and Chen HJ: Roles of circ-CSPP1 on the proliferation and metastasis of glioma cancer. Eur Rev Med Pharmacol Sci 24: 5519-5525, 2020.

22. Klein CA: Cancer progression and the invisible phase of metastatic colonization. Nat Rev Cancer 20: 681-694, 2020.

23. Chan CC, Dostie J, Diem MD, Feng W, Mann M, Rappsilber J and Dreyfuss G: eIF4A3 is a novel component of the exon junction complex. RNA 10: 200-209, 2004.

24. Zhu Y, Ren C and Yang L: Effect of eukaryotic translation initiation factor 4A3 in malignant tumors. Oncol Lett 21: 358, 2021.

25. Lin Y, Zhang J, Cai J, Liang R, Chen G, Qin G, Han X, Yuan C, Liu Z, Li Y, et al: Systematic analysis of gene expression alteration and co-expression network of eukaryotic initiation factor 4A-3 in cancer. J Cancer 9: 4568-4577, 2018.

26. Wang R, Zhang S, Chen X, Li N, Li J, Jia R, Pan Y and Liang H: EIF4A3-induced circular RNA MMP9 (circMMP9) acts as a sponge of miR-124 and promotes glioblastoma multiforme cell tumorigenesis. Mol Cancer 17: 166, 2018.

27. Zheng X, Huang M, Xing L, Yang R, Wang X, Jiang R, Zhang L and Chen J: The circRNA circSEPT9 mediated by E2F1 and EIF4A3 facilitates the carcinogenesis and development of triple-negative breast cancer. Mol Cancer 19: 73, 2020.

cc) (7) $\Theta$ This work is licensed under a Creative Commons Attribution-NonCommercial-NoDerivatives 4.0 International (CC BY-NC-ND 4.0) License. 